\title{
Genehmigungsverfahren klinischer Studien im Bereich der Radioonkologie
}

\author{
Monique Simon · Matthias Habeck · Daniel Büttner · Uta Habeck • \\ Torsten Nölling · Mechthild Krause · Gunnar Brix · Normann Willich • \\ Frederik Wenz $\cdot$ Heinz Schmidberger · Jürgen Debus $\cdot$ Michael Baumann
}

Online publiziert: 26. Oktober 2015

(C) Die Autor(en) 2015. Dieser Artikel ist auf Springerlink.com mit Open Access verfügbar.

\section{Zusammenfassung}

Hintergrund und Ziel Bei Anwendung ionisierender Strahlung in der medizinischen Forschung ist in klinischen Studien im Bereich der Radioonkologie in Deutschland eine

Der vorliegende Artikel ist das Resultat eines von der Deutschen Gesellschaft für Radioonkologie (DEGRO) zusammen mit der Arbeitsgemeinschaft Radioonkologie der Deutschen Krebsgesellschaft (ARO), der Deutschen Gesellschaft für Medizinische Physik (DGMP) und dem vom BMBF etablierten Deutschen Konsortium für Translationale Krebsforschung (DKTK) veranstalteten Workshops zur Klinischen Forschung in der Radioonkologie im Oktober 2013 sowie des nachfolgenden Abstimmungsprozesses mit dem Bundesamt für Strahlenschutz (BfS).

Dieser Aufsatz wurde vom Vorstand der DEGRO am 24.06.2015 als DEGRO-Stellungnahme angenommen.

Monique Simon and Dr. Matthias Habeck share first authorship.

M. Simon $(\bowtie) \cdot$ D. Büttner $\cdot$ M. Krause $\cdot$ J. Debus $\cdot$ M. Baumann Deutsches Konsortium für Translationale Krebsforschung

(DKTK),

Dresden, Deutschland

E-Mail: m.simon@dkfz-heidelberg.de

M. Krause $\cdot$ D. Büttner $\cdot$ M. Simon · M. Baumann

Deutsches Krebsforschungszentrum (DKFZ),

Heidelberg, Deutschland

M. Krause $\cdot$ D. Büttner $\cdot$ M. Simon $\cdot$ M. Baumann

Klinik für Strahlentherapie und Radioonkologie und OncoRay

- Nationales Zentrum für Strahlenforschung in der Onkologie,

Medizinische Fakultät und Universitätsklinikum Carl Gustav

Carus, Technische Universität Dresden,

Fetscherstraße 74,

Dresden 01307, Deutschland

Dr. M. Habeck · U. Habeck · G. Brix

Bundesamt für Strahlenschutz (BfS), Fachbereich Strahlenschutz

und Gesundheit,Ingolstädter Landstraße 1,

Neuherberg 85764, Deutschland

E-Mail: mhabeck@bfs.de
Genehmigung durch das Bundesamt für Strahlenschutz (BfS) notwendig. Bei der Abgrenzung zwischen „Heilkunde" und „medizinischer Forschung" kommt es bei radioonkologischen Studien immer wieder zu widersprüchlichen

T. Nölling

Rechtsanwalt und Fachanwalt für Medizinrecht Torsten Nölling,

Leipzig, Deutschland

M. Krause $\cdot$ M. Baumann

Helmholtz-Zentrum Dresden - Rossendorf, Institut für

Radioonkologie und OncoRay - Nationales Zentrum für

Strahlenforschung in der Onkologie,

Dresden, Deutschland

N. Willich

Klinik für Strahlentherapie - Radioonkologie,

Universitätsklinikum Münster,

Münster, Deutschland

F. Wenz

Klinik für Strahlentherapie und Radioonkologie,

Universitätsmedizin Mannheim, Medizinische Fakultät

Mannheim, Universität Heidelberg,

Mannheim, Deutschland

H. Schmidberger

Universitätsmedizin Mainz, Klinik für Radioonkologie und

Strahlentherapie,

Mainz, Deutschland

J. Debus

Klinik für Radioonkologie und Strahlentherapie,

Universitätsklinikum Heidelberg,

Heidelberg, Deutschland 
Einschätzungen. Ziel ist es, eine Handreichung für Antragsteller, im Beantragungsprozess involvierte Personen und Institutionen sowie Ethikkommissionen zu liefern.

Methoden Die Inhalte sind das Resultat eines von der Deutschen Gesellschaft für Radioonkologie (DEGRO) zusammen mit der Arbeitsgemeinschaft Radioonkologie (ARO) der Deutschen Krebsgesellschaft, der Deutschen Gesellschaft für Medizinische Physik (DGMP) und dem Deutschen Konsortium für Translationale Krebsforschung (DKTK) veranstalten Workshops zur klinischen Forschung in der Radioonkologie im Oktober 2013 sowie des nachfolgenden Abstimmungsprozesses mit dem BfS.

Ergebnisse Die Abgrenzung zwischen Heilkunde und medizinischer Forschung erfolgt anhand der Frage, ob es sich bei der therapeutischen Anwendung um ein nach Art und Umfang klinisch anerkanntes Strahlentherapieverfahren handelt oder nicht. Zur Einordnung werden die bestmögliche Evidenz aus klinischen Studien sowie Stellungnahmen wissenschaftlicher Fachgesellschaften herangezogen. Indikationsstellung, Gesamtdosis und Fraktionierungsschema sind dabei von besonderer Bedeutung. Strahlenanwendungen, die auch außerhalb von Studien eingesetzt werden dürfen, sind nichtgenehmigungsbedürftig.Strahlenanwendungen, die über den geltenden Heilkundestandard hinausgehen, werden als medizinische Forschung klassifiziert und sind genehmigungsbedürftig.

Schlussfolgerung Wichtiges Ziel ist die weitere Professionalisierung der Studienplanung und -durchführung in der Radioonkologie. Eine korrekte Bewertung der Genehmigungsbedürftigkeit einer strahlentherapeutischen Anwendung im Vorfeld eines Forschungsvorhabens verhindertunnötige Kosten und begünstigt eine zeitnahe Bearbeitung der Anträge.

Schlüsselwörter Klinische Forschung ·

StrahlenbehandlungForschung und Entwicklung ·

Genehmigung $\cdot$ Regularien

\section{Approval procedures for clinical trials in the field of radiation oncology}

\begin{abstract}
Background and purpose Application of ionizing radiation for the purpose of medical research in Germany needs to be approved by the national authority for radiation protection (Bundesamt für Strahlenschutz, BfS). For studies in the field of radiation oncology, differentiation between use of radiation for "medical care (Heilkunde)" versus "medical research" frequently leads to contradictions. The aim of this article is to provide principle investigators, individuals, and institutions involved in the process, as well as institutional review or ethics committees, with the necessary information
\end{abstract}

for this assessment. Information on the legal frame and the approval procedures are also provided.

Methods A workshop was co-organized by the German Society for Radiation Oncology (DEGRO), the Working Party for Radiation Oncology (ARO) of the German Cancer Society (DKG), the German Society for Medical Physics (DGMP), and the German Cancer Consortium (DKTK) in October 2013. This paper summarizes the results of the workshop and the follow-up discussions between the organizers and the BfS.

Results Differentiating between "Heilkunde" which does not need to be approved by the BfS and "medical research" is whether the specific application of radiation (beam quality, dose, schedule, target volume, etc.) is a clinically established and recognized procedure. This must be answered by the qualified physician(s) ("fachkundiger Arzt" according to German radiation protection law) in charge of the study and the treatments of the patients within the study, taking into consideration of the best available evidence from clinical studies, guidelines and consensus papers. Among the important parameters for assessment are indication, total dose, and fractionation. Radiation treatments applied outside clinical trials do not require approval by the BfS, even if they are applied within a randomized or nonrandomized clinical trial. The decision-making by the "fachkundigem Arzt" may be supported on request by an opinion given by the DEGRO Expert Committee for clinical trials.

Conclusion An important aim for promoting clinical research and patient care in radiation oncology is to further professionalize planning and implementation of clinical trials in this field. Correct assessment, at an early stage, whether a trial needs to be approved by the BfS may reduce unneccesary costs and reduce the time needed for the approval procedure for those trials which need to be assessed by the BfS.

Keywords Clinical research - Radiation treatments · Research and development $\cdot$ Approval $\cdot$ Regulations

\section{Einführung}

Die Strahlentherapie leistet einen wesentlichen Beitrag zur Versorgung krebskranker Patienten sowie zur Behandlung gutartiger Erkrankungen. Die Patienten ${ }^{1}$ haben dabei Anspruch auf eine Therapie nach wissenschaftlich abgesicherten, modernsten Standards. Diese Standards werden auf Basis retrospektiver und prospektiver klinischer Studien etabliert. Gerade bei lebensbedrohlichen Krebserkrankun-

\footnotetext{
${ }^{1}$ Aufgrund der besseren Lesbarkeit wird der Einfachheit halber nur die männliche Form verwendet. Die weibliche Form ist selbstverständlich immer mit eingeschlossen.
} 
Hier steht eine Anzeige.

Springer 
gen, bei denen die Behandlungsergebnisse heute noch nicht optimal sind, sind weitere Studien zur Verbesserung der Therapie für zukünftige Patienten unverzichtbar und werden von Ärzten, Patienten, ihren Organisationen sowie der Öffentlichkeit nachdrücklich eingefordert. Eine Besonderheit der klinischen Forschung in der Radioonkologie besteht darin, dass die Strahlenanwendungen immer an Patienten und niemals an gesunden Probanden erfolgen.

Ärzte müssen sich vor der Durchführung einer klinischen Studie durch eine unabhängige und interdisziplinär besetzte Ethikkommission beraten lassen [ $\S 15$ Abs. 1 (Muster)-Berufsordnung für Ärzte, MBO-Ä]. Diese berufsrechtliche Pflicht gilt generell für jegliche prospektive klinische Studie und auch für retrospektive Auswertungen klinischer Daten. Beinhaltet das Vorhaben zusätzlich die Anwendung ionisierender Strahlung, ist durch die leitenden Ärzte mit der erforderlichen Fachkunde im Strahlenschutz zu entscheiden, ob die Anwendungen im Rahmen der Heilkunde oder der medizinischen Forschung erfolgen. Sofern es sich bei der geplanten Studie um medizinische Forschung im Sinne des Strahlenschutzrechts handelt, bedarf das Vorhaben der vorherigen Genehmigung durch das Bundesamt für Strahlenschutz (BfS; § 23 Strahlenschutzverordnung, StrlSchV). In $\S 3$ Abs. 2 Nr. 14 StrlSchV ist eine Legaldefinition des Begriffs medizinische Forschung enthalten, die für das Strahlenschutzrecht den Begriff abweichend vom allgemeinen medizinisch-wissenschaftlichen Sprachgebrauch verbindlich definiert. Danach liegt „medizinische Forschung“ im Sinne des $\S 23$ Abs. 1 StrlSchV vor, bei „Anwendung radioaktiver Stoffe oder ionisierender Strahlung am Menschen, soweit sie der Fortentwicklung der Heilkunde oder der medizinischen Wissenschaft und nicht in erster Linie der Untersuchung oder Behandlung des einzelnen Patienten dient.“ Dies ist z. B. bei retrospektiven Studien oder bei prospektiven Studien, die bereits etablierte, dem aktuellen medizinischen Standard entsprechende Behandlungsmethoden miteinander vergleichen, nicht der Fall.

Sowohl bei der Abgrenzung der beiden Anwendungsbereiche „Heilkunde“ und „medizinische Forschung“ als auch bei der Festlegung der Studienkonzepte kommt es bei klinischen Studien in der Radioonkologie immer wieder $\mathrm{zu}$ widersprüchlichen Einschätzungen und somit zu Unsicherheiten, erhöhten Kosten und zeitlichen Verzögerungen bis zum Studienbeginn. Dies steht der allseits formulierten Notwendigkeit für qualitätsgesicherte und von wissenschaftlichen Experten durchgeführte klinische Studien in der Radioonkologie gegenüber. Grundsätzlich besteht die Gefahr, dass neue Bestrahlungstechnologien und Strahlentherapieprotokolle in Deutschland auf breiter Basis unkontrolliert außerhalb von klinischen Studien etabliert werden. Unkontrollierte Implementierungen können ggf. die rechtlichen Vorgaben (fehlendes Vorliegen der rechtfertigenden Indikation gemäß $\S 80$ StrlSchV) missachten und bedin- gen beim Auftreten von Nebenwirkungen oder Versagen der Therapie u. U. auch entsprechende haftungsrechtliche Konsequenzen. Neue Technologien und Schemata brauchen ein qualitätsgesichertes wissenschaftliches Umfeld, um die Patientensicherheit in ausreichendem Maße zu gewährleisten, um die Daten nach wissenschaftlichen Kriterien auszuwerten und um diese für zukünftige Entwicklungen nutzen zu können. Unkontrollierte Implementierungen in der Radioonkologie sind somit weder hinsichtlich des Patienten- und Strahlenschutzes noch bezüglich der Fortentwicklung von Heilkunde und medizinischer Wissenschaft sinnvoll.

Das Ziel dieses Artikels ist es, eine praktische Handreichung für Antragsteller und weitere im Beantragungsprozess für radioonkologische Studien gemäß $§ 15 \mathrm{MBO}-\ddot{A}$ und $\S \S 23,24 \mathrm{StrlSchV}$ involvierte Personen und Institutionen sowie Ethikkommissionen zu liefern. Durch ein genaueres Verständnis des Genehmigungsverfahrens auf Seiten aller Beteiligten kann eine große Zahl von Problemen vermieden werden. Der Artikel wurde von Experten wissenschaftlicher Organisationen der Radioonkologie sowie des BfS gemeinsam verfasst. Er bezieht sich auf Regelungen der StrlSchV ausschließlich zur therapeutischen Anwendung ionisierender Strahlung innerhalb klinischer Studien.

\section{Rechtlicher Rahmen und Verantwortlichkeiten}

Zwei Anwendungsbereiche: Heilkunde vs. medizinische Forschung

$\mathrm{Zu}$ Beginn eines Forschungsvorhabens ist die Entscheidung zu treffen, ob die geplante Strahlenanwendung im Rahmen der Heilkunde oder zum Zweck der medizinischen Forschung erfolgt. Diese Entscheidung liegt in der Verantwortung der Ärzte, welche die Strahlenanwendung leiten und die erforderliche Fachkunde im Strahlenschutz besitzen. Fällt die Strahlenanwendung in den Anwendungsbereich der Heilkunde, haben die fachkundigen Ärzte vor Durchführung der Strahlenanwendung die rechtfertigende Indikation gemäß $\S 80$ StrlSchV zu stellen. Für diese tragen sie die Verantwortung. Bei Strahlenanwendungen zum Zweck der medizinischen Forschung wird hingegen die stets im Einzelfall zu stellende, rechtfertigende Indikation des fachkundigen Arztes durch die für das gesamte Kollektiv der Studienteilnehmer geltende Genehmigung des BfS gemäß $\S 23$ StrlSchV ersetzt. Die erforderliche Abgrenzung zwischen den Anwendungsbereichen Heilkunde und medizinische Forschung stellt sich für den fachkundigen Arzt gelegentlich als problematisch dar und wird dadurch erschwert, dass therapeutische Strahlenanwendungen innerhalb klinischer Studien niemals ausschließlich der Forschung, sondern immer auch der Krankenversorgung dienen. 
Entscheidend für die Abgrenzung zwischen nichtgenehmigungsbedürftiger Heilkunde und genehmigungsbedürftiger medizinischer Forschung im Sinne der StrlSchV ist die Frage, ob es sich bei der therapeutischen Anwendung um ein nach Art und Umfang klinisch anerkanntes Strahlentherapieverfahren handelt oder nicht. Dabei sind u. a. die Indikationsstellung für den Therapieansatz, die Gesamtdosis und das Fraktionierungsschema von besonderer Bedeutung. Ein Therapieverfahren gilt als anerkannt, wenn es dem Stand der Heilkunde entspricht. Die Einordnung als klinisch anerkanntes Therapieverfahren erfolgt dabei anhand der aktuell zusammengefassten bestmöglichen Evidenz aus klinischen Studien (Originalpublikationen als Primärliteratur, Übersichtsarbeiten und Standardlehrbücher als Sekundärliteratur) und anhand von nationalen oder internationalen Stellungnahmen der zuständigen wissenschaftlichen Fachgesellschaften (z. B. Leitlinien). Kommen im Rahmen einer Studie ausschließlich Strahlenanwendungen zum Einsatz, die anhand dieser Kriterien nach Art und Umfang auch außerhalb der Studie Anwendung finden dürfen, sind diese unter Strahlenschutzgesichtspunkten als nichtgenehmigungsbedürftige Heilkunde einzustufen. Dies gilt auch dann, wenn mehrere etablierte Strahlenanwendungen im Rahmen einer Studie miteinander verglichen werden sollen und hierzu die Patienten randomisiert auf die beiden Studienarme verteilt werden. Der fachkundige Arzt muss in diesen Fällen für jeden einzelnen Patienten entsprechend dieser Kriterien die rechtfertigende Indikation unter Abwägung von Nutzen und Risiko stellen. Im Gegensatz dazu gelten Strahlenanwendungen im Rahmen einer Studie, die nach Art und/oder Umfang über den geltenden Heilkundestandard hinausgehen, als medizinische Forschung und sind zur Genehmigung beim BfS einzureichen.

Häufig wird die Frage gestellt, ob die Berücksichtigung moderner biologischer Bildgebungsverfahren bei der Bestrahlungsplanung einen Einfluss auf die Genehmigungsbedürftigkeit der strahlentherapeutischen Anwendung selbst hat. Solange es sich bei einer strahlentherapeutischen Anwendung um ein klinisch anerkanntes Strahlentherapieverfahren im o. g. Sinne handelt, kann jede auf Basis der wissenschaftlichen Evidenz zur Festlegung des Zielvolumens etablierte Bildgebungsmethode für die Bestrahlungsplanung herangezogen werden, ohne dass daraus die Genehmigungsbedürftigkeit der strahlentherapeutischen Anwendung selbst resultieren würde.

Für die Kommunikation zwischen fachkundigem Arzt, wissenschaftlichen Fachgesellschaften, Ethikkommissionen und dem BfS ist die Verwendung einer klaren Terminologie von immenser Bedeutung. Als Beispiel eines häufig missverstandenen Begriffs sei hier der des „Therapiestandards“ angeführt. Oft wird das Wirtschaftlichkeitsgebot des SGB V fälschlicherweise mit dem medizinischen Standard in Verbindung gebracht. Für den Bereich der Strahlentherapie gilt:
Ein Standardverfahren im Sinne der StrlSchV meint jedoch ein im Rahmen der regulären Krankenversorgung (Heilkunde) angewendetes Behandlungsverfahren, für das eine rechtfertigende Indikation durch einen fachkundigen Arzt gestellt werden kann, wobei dies nicht die Wirtschaftlichkeit und somit die Erstattung durch die Kostenträger impliziert. Im Rahmen einer Studie können durchaus Behandlungskonzepte geprüft werden, zu denen in medizinischer Hinsicht ausreichende Erkenntnisse vorliegen, um sie im Sinne des Strahlenschutzes als Heilkunde einzuschätzen, die aber von den Kostenträgern (noch) nicht als zweckmäßig und wirtschaftlich eingeschätzt und somit nicht erstattet werden. Deshalb ist es notwendig, bei Empfehlungen und Leitlinien wissenschaftlicher Fachgesellschaften zu konkretisieren, in welchem Zusammenhang der Begriff „Standardtherapie“ gebraucht wird (z. B. durch entsprechende Disclaimer).

Es ist aufgrund der Erfahrungen der letzten Jahre wichtig, darauf hinzuweisen, dass für die Abgrenzung zwischen Heilkunde und genehmigungsbedürftiger medizinischer Forschung Merkmale der Studie, wie z. B. die Größe und Bedeutung, die Zahl der teilnehmenden Zentren und das Studiendesign (z. B. kontrolliert oder nichtkontrolliert, randomisiert oder nichtrandomisiert), im strahlenschutzrechtlichen Sinne keine Entscheidungskriterien sind.

Im Falle neuartiger Strahlenanwendungen, bei denen Nutzen und Risiko für den Patienten noch unbekannt sind, kann die für die Stellung der rechtfertigenden Indikation notwendige individuelle Nutzen-Risiko-Abwägung nicht vorgenommen werden, so dass es sich - sofern nicht ein individueller Heilversuch vorliegt - nicht um Heilkunde handelt. Konkrete Beispiele für die Abgrenzung zwischen Heilkunde und genehmigungsbedürftiger medizinischer Forschung sind in Tab. 1 aufgeführt.

\section{Verantwortlichkeit bei der Durchführung einer Strahlenanwendung}

Unabhängig von der Frage, ob eine Strahlenanwendung im Rahmen der Heilkunde mit der rechtfertigenden Indikation eines fachkundigen Arztes oder im Rahmen der medizinischen Forschung mit der Genehmigung des BfS durchgeführt wird, trägt der die jeweilige Anwendung leitende Arzt die Verantwortung für die Durchführung der Strahlenanwendung. Eine Verlagerung dieser Verantwortlichkeit auf andere Stellen (z. B. auf die Genehmigungsbehörde) ist ausgeschlossen. Gemäß der in der Muster-Berufsordnung für Ärzte verankerten Deklaration von Helsinki des Weltärztebundes (WMA) haben die an einem Forschungsvorhaben beteiligten Ärzte dafür Sorge zu tragen, dass „Maßnahmen zur Risikominimierung [...] implementiert werden. Die Risiken müssen vom Forscher kontinuierlich überwacht, eingeschätzt und dokumentiert werden. [...] Sobald sich herausstellt, dass die Risiken den potentiellen Nutzen über- 
Tab. 1 Beispiele für die Abgrenzung von Heilkunde und genehmigungsbedürftiger medizinischer Forschung (Stand 2015)

\begin{tabular}{|c|c|c|c|c|}
\hline Beispiel & $\begin{array}{l}\text { Randomisiertes } \\
\text { Studiendesign? }\end{array}$ & Studiendesign & $\begin{array}{l}\text { Primärzweck: Heilkun- } \\
\text { de oder Forschung? }\end{array}$ & Anmerkungen \\
\hline $\begin{array}{l}\text { Evaluation von } 4 \text { Behandlungs- } \\
\text { modalitäten zur Therapie des } \\
\text { Prostatakarzinoms mit niedri- } \\
\text { gem oder ,frühem intermediä- } \\
\text { ren“ Risiko (PREFERE-Studie) }\end{array}$ & $\mathrm{Ja}$ & $\begin{array}{l}4 \text { Therapieoptionen: } \\
\text { - Radikale Prostatektomie } \\
\text { - Perkutane Radiotherapie } \\
\text { - Permanente } \\
\text { Seed-Implantation } \\
\text { - Active Surveillance }\end{array}$ & Heilkunde & $\begin{array}{l}\text { Die } 4 \text { Behandlungsschema- } \\
\text { ta sind etabliert, an großen } \\
\text { Patientenkollektiven einzeln } \\
\text { evaluiert und werden in der } \\
\text { regulären Krankenversorgung } \\
\text { nebeneinander eingesetzt, } \\
\text { obwohl keine randomisierten } \\
\text { Studien zum direkten Ver- } \\
\text { gleich vorliegen. }\end{array}$ \\
\hline $\begin{array}{l}\text { Neoadjuvante Bestrahlung } \\
\text { des lokal fortgeschrittenen } \\
\text { Rektumkarzinoms }\end{array}$ & $\mathrm{Ja}$ & $\begin{array}{l}\text { Photonen } 25 \mathrm{~Gy} ; 5 \mathrm{~Gy} / \text { Frak- } \\
\text { tion; } 5 \text { Fraktionen/Woche } \\
\text { vs. } \\
\text { Photonen } 50 \mathrm{~Gy} ; 2 \text { Gy/Frak- }\end{array}$ & Heilkunde & $\begin{array}{l}\text { Beide Verfahren sind etabliert } \\
\text { und werden in der regulären } \\
\text { Krankenversorgung nebenein- } \\
\text { ander eingesetzt. }\end{array}$ \\
\hline
\end{tabular}

Primäre definitive Bestrahlung Ja Photonen 76 Gy; 2 Gy/Frak- Heilkunde des lokal fortgeschrittenen tion; 5 Fraktionen/Woche vs. Prostatakarzinoms

Protonen 76 Gy (RBE); 2 Gy (RBE) pro Fraktion; 5 Fraktionen/Woche

Prätherapeutische $\left[{ }^{11} \mathrm{C}\right]-\mathrm{Me}$ thionin-PET als Biomarker für Zeitpunkt und Ort von Glioblastom-Rezidiven

Phase-III-Studie zur kombinierten Radiochemotherapie des lokal fortgeschrittenen HNSCC

Phase-II-Dosiseskalationsstudie Nein zur Behandlung des lokal fortgeschrittenen HNSCC

Phase-III-Studie beim Mammakarzinom

Phase-II-Studie zur postoperativen Bestrahlung des Glioblastoms in der Primärsituation (ohne Vorbestrahlung)
Nein Ja

Photonen 70 Gy; 2 Gy/Fraktion; 5 Fraktionen/Woche über 7 Wochen plus Cisplatin vs. Photonen 70 Gy; 2 Gy/Fraktion; 5 Fraktionen/Woche, über 7 Wochen plus Cetuximab Basierend auf Biomarkern bei Hochrisikopatienten Dosiseskalation von $70 \mathrm{~Gy}$ auf bis zu 80 Gy in 6 Wochen

Photonen 60 Gy; 2 Gy/Fraktion; 5 Fraktionen/Woche plus tgl. Temozolomid $\left(75 \mathrm{mg} / \mathrm{m}^{2}\right.$ Körperoberfläche p. o.)

Heilkunde

Heilkunde

Forschung

Photonen 50 Gy plus 16 Gy Forschung

Boost; 2 Gy/Fraktion;

5 Fraktionen/Woche

vs.

Kohlenstoffionen 50 Gy (RBE)

plus 16 Gy (RBE) Boost;

2 Gy (RBE) pro Fraktion;

5 Fraktionen/Woche

Photonen 60 Gy; 2 Gy/Fraktion; 5 Fraktionen/Woche über 6 Wochen plus Temozolomid vs.

Photonenstereotaxie 32,5 Gy;

6,5 Gy/Fraktion; 5 Fraktionen/

Woche über 1 Woche plus

Temozolomid

Forschung
Bei gleicher Dosis und Fraktionierung hat der Vergleich der Ergebnisse verschiedener Studien zu Photonen bzw. Protonen mit großen Patientenzahlen keine Unterschiede gezeigt. Beide Verfahren werden nebeneinander eingesetzt.

Sowohl das Strahlentherapieverfahren als auch die PETMethode sind etabliert. Das strahlentherapeutische Vorgehen wird durch die Ergebnisse der PET-Untersuchung nicht beeinflusst.

Zwei unabhängig voneinander etablierte Standardbehandlungskonzepte werden im Rahmen der Studie miteinander verglichen.

Es fehlen hinsichtlich des dosiseskalierten Behandlungskonzeptes bisher ausreichende Erkenntnisse, um die für die Stellung der rechtfertigenden Indikation erforderliche Nutzen-Risiko-Abwägung vornehmen zu können.

Es fehlen hinsichtlich der Kohlenstoffionentherapie bisher ausreichende Erkenntnisse, um die für die Stellung der rechtfertigenden Indikation erforderliche Nutzen-RisikoAbwägung vornehmen zu können.

Es fehlen hinsichtlich des hypofraktionierten Behandlungskonzeptes bisher ausreichende Erkenntnisse, um die für die Stellung der rechtfertigenden Indikation erforderliche Nutzen-Risiko-Abwägung vornehmen zu können. 
steigen, oder wenn es einen schlüssigen Beweis für gesicherte Erkenntnisse gibt, müssen Ärzte einschätzen, ob die Studie fortgesetzt, modifiziert oder unverzüglich beendet werden muss.“ [1].

\section{Individueller Heilversuch}

Eine Sonderstellung nimmt der individuelle Heilversuch ein, der aus dem Bereich des ärztlichen Berufsrechts stammt und nicht strahlenschutzrechtlich normiert ist. Dieser dient primär der Heilung, Erkennung, Verhütung oder Linderung einer Krankheit oder eines Leidens eines individuellen Patienten. Im Gegensatz zur Heilkunde werden hier jedoch mangels anderer erfolgversprechender Methoden noch nicht voll erprobte Verfahren mit dem konkreten Ziel einer individuellen Heilmaßnahme angewendet. Durch diesen auf den individuellen Patienten gerichteten Zweck unterscheidet sich der Heilversuch von einer wissenschaftlichen Studie [2]. Der individuelle Heilversuch sieht keinen Erkenntnisgewinn über den Einzelfall hinaus vor, d. h. ihm liegen kein wissenschaftlicher Prüfplan und auch keine Absicht zur Änderung des Therapiestandards zugrunde. Für den individuellen Heilversuch ist weder die Zustimmung der Ethikkommission noch die des BfS erforderlich. Der individuelle Heilversuch liegt im Ermessen und in der Verantwortung des fachkundigen Arztes, der zusammen mit dem Patienten die Entscheidung hierzu trifft. An die Dokumentation der Entscheidungsfindung für die Durchführung eines individuellen Heilversuchs anhand wissenschaftlicher Kriterien sowie an die Aufklärung des Patienten über die Natur der Behandlung als individueller Heilversuch sind besonders hohe Maßstäbe anzulegen. Aus dem auf den individuellen Patienten gerichteten und zu begründenden Zweck des individuellen Heilversuchs geht hervor, dass dieser weder systematisch (quasi als Ersatz für klinische Studien an Patientenkollektiven) noch als Standardtherapie eingesetzt werden darf.

\section{Fachkundiger Arzt}

Der für die Strahlentherapie fachkundige Arzt ist in $\S 30$ der StrlSchV definiert. Er muss seine Fachkunde durch eine entsprechende Fachkundebescheinigung nachweisen und alle fünf Jahre aktualisieren. Der fachkundige Arzt entscheidet, ob die geplante Strahlenanwendung im Rahmen der Heilkunde erfolgt oder als medizinische Forschung einzustufen ist. Bei multizentrischen Studien muss an jedem Standort mindestens ein fachkundiger Arzt für die Studie zur Verfügung stehen, der die Verantwortung für die Durchführung der Strahlenanwendungen trägt.

\section{DEGRO-Expertengremium}

Sollte die Abgrenzung Heilkunde versus medizinische Forschung dem fachkundigen Arzt Schwierigkeiten bereiten, kann das unabhängige Expertengremium der Deutschen Gesellschaft für Radioonkologie (DEGRO) hinzugezogen werden, welches in Zusammenarbeit mit dem BfS eingerichtet wurde. Das Expertengremium der DEGRO setzt sich aus ausgewiesenen Experten auf dem Gebiet der Radioonkologie und Strahlentherapie zusammen. Anhand eines Formblatts (http://www.degro.org/dav/html/download/doc/ExpertengremiumVoranfrage.doc), das der fachkundige Arzt und Gesamtstudienleiter für Deutschland ausgefüllt hat, bewerten die Mitglieder des Expertengremiums, ob eine therapeutische Strahlenanwendung in den Anwendungsbereich der Heilkunde fällt oder ob es sich um genehmigungsbedürftige medizinische Forschung handelt. Anhand der schriftlichen Stellungnahme des Gremiums entscheidet letztendlich der fachkundige Arzt, ob die geplante Maßnahme als Heilkunde einzustufen ist. Er ist für diese Entscheidung verantwortlich. Bei multizentrischen Studien gilt dies analog auch für die fachkundigen Ärzte an den einzelnen Studienzentren. Hat der jeweilige fachkundige Arzt entschieden, dass die geplante Therapie als Heilkunde einzuschätzen ist, ist keine weitere Einbindung des BfS notwendig. Die Entscheidung ist unter Ausführung der maßgeblichen Gründe sorgfältig zu dokumentieren und die Dokumentation zu archivieren.

Die Autoren dieses Artikels empfehlen den Studienleitern, von der Möglichkeit einer Begutachtung durch das DEGRO-Expertengremium großzügig Gebrauch zu machen und bei bestehenden Zweifeln an der Einstufbarkeit als Heilkunde die Studie beim BfS zur Genehmigung einzureichen. Auch wenn die Stellungnahme des Expertengremiums rechtlich nicht bindend ist und die alleinige Verantwortung letztendlich bei den fachkundigen Ärzten an den einzelnen Studienzentren verbleibt, kann die Stellungnahme z. B. bei späteren Streitfällen von hoher Relevanz sein und zur Absicherung der Entscheidungsträger beitragen.

\section{Ethikkommission}

Jegliche medizinische Studie, ob retrospektiv oder prospektiv, obliegt laut Berufsrecht der Ärzte der Beratungspflicht durch die zuständige Ethikkommission ( $(15 \mathrm{MBO}-\mathrm{A})$. Der fachkundige Arzt muss vor der Einreichung der Studie bei der Ethikkommission entschieden haben, ob die geplante Strahlenanwendung im strahlenschutzrechtlichen Sinne in den Anwendungsbereich der Heilkunde oder der medizinischen Forschung fällt. Die Entscheidung muss er der Ethikkommission mitteilen. Bei Einreichung eines Vorhabens, das der fachkundige Arzt als Heilkunde eingeordnet hat, berät die Ethikkommission das Forschungsvorhaben nach 
Tab. 2 Genehmigungsvoraussetzungen für Anwendungen ionisierender Strahlung zum Zweck der medizinischen Forschung (§ 24 Abs. 1 StrlSchV)

1. Für das beantragte Forschungsvorhaben muss ein zwingendes Bedürfnis bestehen, begründet darin, dass die bisherigen Forschungsergebnisse und die medizinischen Erkenntnisse nicht ausreichen.

2. Die Anwendung ionisierender Strahlung kann nicht durch eine andere (Untersuchungs- oder) Behandlungsart ersetzt werden, die keine Strahlenexposition verursacht.

3. Die strahlenbedingten Risiken, die mit der Strahlenanwendung für den Patienten verbunden sind, gemessen an der voraussichtlichen Bedeutung der Ergebnisse für die Fortentwicklung der Heilkunde oder der medizinischen Wissenschaft, sind ärztlich gerechtfertigt.

4. Die für die medizinische Forschung vorgesehenen Anwendungsarten ionisierender Strahlung entsprechen dem Zweck der Forschung und können nicht durch andere Anwendungsarten ionisierender Strahlung ersetzt werden, die zu einer geringeren Strahlenexposition für den Patienten führen würden.

5. Die bei der Anwendung ionisierender Strahlung auftretende Strahlenexposition kann nach dem Stand von Wissenschaft und Technik nicht weiter herabgesetzt werden, ohne den Zweck des Forschungsvorhabens zu gefährden.

6. (Die Körperdosis des Patienten wurde abgeschätzt.)

Anmerkung der Autoren: Die Abschätzung der Körperdosis ist bei teletherapeutischen Strahlenanwendungen wenig zweckmäßig. Daher wird in den Formblättern des BfS nach der Dosis in den Zielvolumina und Risikoorganen sowie nach den der Therapieplanung zugrunde gelegten ,,dose constraints" gefragt.

7. Die Anzahl der Patienten ist auf das notwendige Maß beschränkt.

8. Die Stellungnahme einer Ethikkommission zu dem beantragten Forschungsvorhaben liegt vor ( $92 \mathrm{StrlSchV})$.

9. Die Strahlenanwendung wird von einem Arzt geleitet, der eine mindestens 2-jährige Erfahrung in der Anwendung ionisierender Strahlung am Menschen nachweisen kann, der die erforderliche Fachkunde im Strahlenschutz besitzt und während der Anwendung ständig erreichbar ist. Bei der Planung und bei der Anwendung wird ein Medizinphysikexperte hinzugezogen.

10. Die erforderliche Vorsorge für die Erfüllung gesetzlicher Schadensersatzverpflichtungen wurde getroffen.

11. Es liegt eine Genehmigung für den Betrieb von Anlagen zur Erzeugung ionisierender Strahlung vor.

ethischen und berufsrechtlichen Gesichtspunkten und gibt hierzu ein schriftliches Votum ab. Bei Einreichung eines Vorhabens, das vom fachkundigen Arzt als genehmigungsbedürftige medizinische Forschung eingeschätzt worden ist, muss die beim BfS registrierte Ethikkommission ihr schriftliches Votum innerhalb von 60 Tagen nach Eingang der erforderlichen Unterlagen abgeben. Dabei muss sie neben der Gesamtbewertung insbesondere darauf eingehen, ob für das beantragte Vorhaben ein zwingendes Bedürfnis besteht. Ein solches zwingendes Bedürfnis ist gegeben, wenn die bisherigen Forschungsergebnisse und die medizinischen Erkenntnisse nicht ausreichend sind $(\S 24$ Abs. 1 Nr. 1 StrlSchV). Bei multizentrischen Studien reicht die Bestätigung des zwingenden Bedürfnisses durch eine einzige Ethikkommission aus.

\section{Bundesamt für Strahlenschutz}

Der fachkundige Arzt trifft mit oder ohne vorherige schriftliche Stellungnahme des DEGRO-Expertengremiums die Entscheidung, ob er für die geplanten Strahlenanwendungen die rechtfertigende Indikation stellen kann. Sofern er dies nicht kann, muss er das Vorhaben dem BfS zur Genehmigung der Strahlenanwendungen gemäß § 23 StrlSchV vorlegen. In diesem Fall müssen formalisierte Unterlagen eingereicht werden, die weiter unten aufgeführt sind. Auch wenn das BfS die Studie genehmigt, liegt die Verantwortung für die Durchführung der Strahlenanwendungen beim einzelnen fachkundigen Arzt am jeweiligen Studienzentrum.

\section{Erforderliche Voraussetzungen genehmigungspflichtiger Studien}

Die Genehmigungsvoraussetzungen für die Anwendung ionisierender Strahlung in der medizinischen Forschung sind in $\S 24$ Abs. 1 StrlSchV geregelt (Tab. 2). Das BfS stellt für Studien, die zur Genehmigung eingereicht werden, Hinweise und Formblätter zur Verfügung (http://www.bfs.de/ $\mathrm{DE} /$ themen/ion/anwendung-medizin/forschung/formblaetter/formblaetter.html):

- Für die Beantragung therapeutischer Strahlenanwendungen ist immer ein ausführliches Genehmigungsverfahren (§ 24 Abs. 1 StrlSchV) und nicht das vereinfachte Verfahren (§ 24 Abs. 2 StrlSchV, sog. Fallgruppe „Begleitdiagnostik“) erforderlich. Ist neben der in der regulären Krankenversorgung für die Strahlentherapie dieser Erkrankung etablierten Standarddiagnostik noch eine studienbedingte Begleitdiagnostik unter Anwendung ionisierender Strahlung vorgesehen, muss diese zusätzlich beantragt werden (derzeit Formblatt A).

- Die personellen und sachlichen Voraussetzungen müssen (bei multizentrischen Studien für jedes Studienzentrum einzeln) nachgewiesen und vom Strahlenschutzverantwortlichen bestätigt werden (derzeit Formblatt B).

- Sämtliche in Deutschland teilnehmenden Studienzentren sind aufzuführen (derzeit Formblatt C).

- Die medizinisch-wissenschaftlichen Voraussetzungen für die Strahlenanwendungen sind nachvollziehbar darzulegen (derzeit Formblatt D). 
- Beizufügen sind ebenfalls der Nachweis über die atomrechtliche Deckungsvorsorge (z. B. Strahlen-Haftpflichtversicherung) und die Stellungnahme einer beim BfS registrierten Ethikkommission einschließlich der Bestätigung des zwingenden Bedürfnisses für das Forschungsvorhaben gemäß $§ 24$ Abs. 1 Nr. 1 StrlSchV.

Wenn es sich bei der vorgesehenen Studie gleichzeitig um eine klinische Prüfung nach AMG/MPG handelt (z. B. bei Prüfung der therapeutischen Strahlenanwendung in Kombination mit einem neuen Arzneimittel), ist die Studie sowohl beim BfS als auch bei der jeweils weiteren, überdies zuständigen Bundesoberbehörde (Bundesinstitut für Arzneimittel und Medizinprodukte (BfArM) oder Paul-Ehrlich-Institut (PEI)) zur Genehmigung vorzulegen.

\section{Bewertung strahlentherapeutischer Studienanträge durch das BfS}

Der Gesetzgeber hat in der StrlSchV festgelegt, dass eine Anwendung ionisierender Strahlung oder radioaktiver Stoffe zum Zweck der medizinischen Forschung einer Genehmigung durch das Bundesamt für Strahlenschutz bedarf ( $§ 23 \mathrm{StrlSchV})$. Ziel des Prüfauftrags der Genehmigungsbehörde ist der Schutz der Studienteilnehmer vor schädlichen Auswirkungen der Strahlenanwendung. Ein zentraler Punkt im Rahmen des Genehmigungsverfahrens ist daher die Frage, ob die mit der Anwendung für den Studienteilnehmer verbundenen Risiken ärztlich gerechtfertigt sind (vgl. § 24 Abs. 1 Nr. 3 StrlSchV). Folgende Besonderheiten strahlentherapeutischer Studien erschweren u. a. diese Bewertung:

- Die Patienten, die an strahlentherapeutischen Studien teilnehmen, sind in der Regel schwer erkrankt, haben aber angesichts der oft kurativen Intention des zu prüfenden Behandlungskonzepts eine Chance auf eine dauerhafte Tumorheilung und damit auf ein Langzeitüberleben. Hier besteht ein wichtiger Unterschied zu vielen Arzneimittelstudien in der Onkologie, die inkurable Patienten rekrutieren.

- Bei kurativen Behandlungskonzepten können höhere Risiken gerechtfertigt sein (z. B. in Form einer gegenüber dem konventionellen Behandlungskonzept gesteigerten Nebenwirkungsrate), wenn sich dadurch bessere Heilungschancen bieten.

- Die Abschätzung der potentiellen Risiken eines neuen Behandlungskonzepts kann in der Regel am besten durch Vergleich mit bereits evaluierten/etablierten Behandlungskonzepten erfolgen. Dieser Vergleich wird jedoch dadurch erschwert, dass im Rahmen von Studien bezüglich Fraktionierungsschema und Strahlenquali- tät z. T. von gängigen Behandlungskonzepten abgewichen wird. Standards für die Dosisspezifikation und die Konturierung der Zielvolumina können heterogen sein. Daher und aufgrund der unterschiedlichen Patientenanatomie, Tumorlokalisationen und -ausdehnungen können Dosisverteilungen interindividuell z. T. höchst unterschiedlich ausfallen. Die geplante Zielvolumendosis allein spezifiziert deshalb das Behandlungskonzept (anders als die Dosis einer medikamentösen Therapie) nur unzureichend.

An die Qualität strahlentherapeutischer Studien sind dabei strenge Anforderungen zu stellen. Zur Beurteilung radioonkologischer Studienanträge sind Parameter heranzuziehen, die die größtmögliche Sicherheit für die Patienten sowie qualitativ hochwertige Studienergebnisse gewährleisten, ohne einen Fortschritt bei der Behandlung von Krebserkrankungen unangemessen zu verhindern. Im folgenden Abschnitt werden einige besonders wichtige Prüfpositionen bei der Bewertung strahlentherapeutischer Studien näher erläutert.

\section{Studiendesign}

Bei einem neuen Behandlungskonzept mit noch unbekannter Nutzen-Risiko-Relation müssen besondere Ansprüche an das zugrundeliegende Design der Studie gestellt werden. Die zentrale Frage bei der Bewertung solcher Konzepte lautet: Ist die im Rahmen der Studie vorgesehene Therapie unter Berücksichtigung des gewählten Studiendesigns ärztlich vertretbar? Durch den Antragsteller ist in diesem Zusammenhang ausführlich zu begründen, dass der Patient durch seine Studienteilnahme keinem unvertretbar hohen Risiko ausgesetzt wird. Dabei ist der Genehmigungsbehörde insbesondere ausführlich darzulegen, ob Ergebnisse bezüglich Sicherheit und Wirksamkeit von Vorgängerstudien mit ähnlicher Fragestellung und ggf. vergleichbarer Zielvolumendosis existieren. In diesem Zusammenhang kann auch die Angabe der biologisch äquivalenten Dosis in Dosen pro Fraktion von 2 Gy (EQD2) hilfreich sein, d. h. diejenige Dosis, die der zu verschreibenden Gesamtdosis voraussichtlich entspräche, wenn sie in konventioneller Fraktionierung mit ultraharter Röntgenstrahlung (oder Elektronen) appliziert würde. Je nach Behandlungskonzept kann die Angabe der EQD2 mit mehr oder weniger großen Unsicherheiten verbunden sein. Dennoch gibt die diesbezügliche Abschätzung des Antragstellers der Genehmigungsbehörde Anhaltspunkte für die Einordnung des beantragten Behandlungskonzepts.

Das Studiendesign ist so $\mathrm{zu}$ wählen, dass potentielle Risiken für einzelne Studienteilnehmer nach Möglichkeit minimiert werden. Das Auftreten von Nebenwirkungen, die hinsichtlich ihrer Art, Häufigkeit und/oder ihres Schwere- 
grads als inakzeptabel anzusehen sind, ist im Vorfeld einer strahlentherapeutischen Studie nicht vollständig auszuschließen. Aus Sicht der Genehmigungsbehörde muss aber bei neuen Behandlungskonzepten gewährleistet werden, dass auftretende inakzeptable Nebenwirkungen auf eine möglichst geringe Zahl von Studienteilnehmern beschränkt bleiben. Die Beachtung einiger Grundsätze bereits bei der Konzeption der Studie kann aus Sicht der Genehmigungsbehörde zur Erhöhung der Sicherheit der Studienteilnehmer beitragen:

- Grundsätzlich sollten bei noch unklarer Nutzen-RisikoRelation nur wenige Patienten zeitgleich mit dem experimentellen Konzept behandelt werden. Erfahrungen aus der Behandlung der ersten Patienten können dann (z. B. im Rahmen von Zwischenauswertungen unter Wahrung einer angemessenen Nachbeobachtungszeit) vor der Rekrutierung weiterer Patienten in eine aktualisierte Bewertung der Nutzen-Risiko-Relation einfließen.

- Sobald nähere Erkenntnisse bezüglich Sicherheit und Wirksamkeit vorliegen, können ggf. Nachfolgestudien mit größerer Teilnehmerzahl geplant werden.

- Bei einer deutlichen Anhebung der Gesamtdosis über den etablierten Heilkundestandard hinaus sollte die Dosiseskalation schrittweise vorgenommen werden.

- Der Therapieplanung sollten nach Möglichkeit „dose constraints“ für Risikoorgane zugrundegelegt werden, deren Sicherheit durch entsprechende Literaturstellen gut belegt ist.

- Vor Studienbeginn sollten Abbruchregeln (,stopping rules") definiert werden. Bei ihrer Festlegung ist zu beachten, dass sie möglichst keinen Abbruch der Studie aufgrund von Akuttoxizitäten erzwingen, die angesichts erst später beurteilbarer, u. U. deutlich verbesserter Heilungschancen noch gerechtfertigt sein könnten.

- Die Ein- und Ausschlusskriterien der Studie sollten so formuliert werden, dass Patienten mit einem zu hohen Risiko für inakzeptable Nebenwirkungen zunächst von der Studienteilnahme ausgeschlossen bleiben. $\mathrm{Zu}$ einem späteren Zeitpunkt kann bei erwiesener guter Wirksamkeit und Verträglichkeit des Behandlungskonzepts das Patientenkollektiv erweitert werden.

Bezüglich der Methodologie strahlentherapeutischer Studien gibt es bisher keinen ausreichenden Konsens [3]. Das klassische ,3+3“-Design ist für die Evaluation neuer radio(chemo)therapeutischer Behandlungskonzepte angesichts der teilweise langen Latenzzeiten bis zum Auftreten relevanter Toxizitäten nicht sonderlich gut geeignet. Biostatistische Methoden, die nicht nur aufgetretene Akuttoxizitäten, sondern auch Spättoxizitäten in das Rekrutierungsschema einbeziehen, sind aufwändiger und werden angesichts ihrer geringeren Praktikabilität in der Praxis bisher kaum eingesetzt. Dabei sind es gerade die Spättoxizi- täten, die Gesundheit und Lebensqualität der behandelten Patienten langfristig beeinträchtigen können. Im Rahmen strahlentherapeutischer Studien ist deshalb eine breitere Evaluierung von neueren statistischen Verfahren wünschenswert (z. B. „time-to-event continual reassessment method", TITE-CRM [4]).

\section{Patienteninformation}

Wie jede ärztliche Behandlung darf eine Strahlentherapie nur nach vorheriger Aufklärung über Risiken und Erfolgsaussichten und nach wirksamer Einwilligung des Patienten erfolgen. Wenn bei einem neuen Behandlungskonzept, das im Rahmen einer strahlentherapeutischen Studie evaluiert werden soll, nennenswerte Unsicherheiten im Hinblick auf die erhoffte therapeutische Wirkung oder auf die Art, den Schweregrad und die Häufigkeit der Nebenwirkungen bestehen, muss die Patienteninformation besonders hohen Anforderungen genügen. Der Patient ist darüber aufzuklären, dass es sich bei der geplanten Therapie um ein bisher nicht etabliertes Konzept mit noch ungeklärter Nutzen-Risiko-Relation handelt, das sich langfristig als der Standardtherapie überlegen, gleichwertig oder im ungünstigen Falle auch unterlegen erweisen könnte. Vor diesem Hintergrund ist der Patient besonders sorgfältig über bereits im Rahmen der regulären Krankenversorgung etablierte Behandlungskonzepte (einschließlich der damit verbundenen potentiellen Vor- und Nachteile) aufzuklären.

\section{Nachsorge}

Wie bereits oben ausgeführt, kann die Bewertung von Nutzen und Risiko eines neuartigen strahlentherapeutischen Konzepts nur auf der Grundlage einer langfristigen Nachbeobachtung der Studienpatienten erfolgen. Die Durchführung regelmäßiger Nachsorgeuntersuchungen ist daher von grundlegender Bedeutung. Die von der Strahlenschutzkommission (SSK) im Jahre 1998 geforderte Dauer der Nachsorge beträgt 5 Jahre im Rahmen der Heilkunde [5]. Die aktuellen Empfehlungen der SSK zur Nachsorge in der Strahlentherapie [6] gehen sogar weit darüber hinaus: Demnach ,ist eine Beschränkung des Nachuntersuchungszeitraums auf 5 Jahre nicht gerechtfertigt", da Nebenwirkungen der Strahlentherapie auch noch deutlich später zutage treten können. Bei genehmigungsbedürftigen Studien kann der geforderte Nachsorgezeitraum allerdings nicht immer im Studienprotokoll festgeschrieben werden, da nach den bisherigen Erfahrungen der Autoren bei Abschluss der gemäß $\S 24$ Abs. 1 Nr. 10 StrlSchV erforderlichen Vorsorge für die Erfüllung gesetzlicher Schadensersatzverpflichtungen von den Versicherungsunternehmen gefordert wird, die Dauer der Studie inklusive Nachbeobachtungszeitraum auf maximal 10 Jahre zu begrenzen. Insofern muss die Nachsorge 
in eine Nachbeobachtung innerhalb des Studienprotokolls (maximal 10 Jahre für Rekrutierung und Nachbeobachtung) und eine darüber hinausgehende langfristige Nachsorge aus Sicht des Strahlenschutzes getrennt werden, die nicht Bestandteil des Studienprotokolls sein kann. Diese sollte von DEGRO und BfS gemeinsam in Übereinstimmung mit der SSK definiert werden.

Weiter heißt es in der Stellungnahme der SSK: „Patienten mit hohem Risiko für unerwünschte Strahlenfolgen, z. B. nach Behandlung mit neu eingeführten Therapiestrategien, bei Anwendung von dosiseskalierten Protokollen oder mit aggressiver Radiochemotherapie [...], müssen in der Einrichtung selbst nachuntersucht werden. Nur durch die Kompetenz der behandelnden Ärzte ist gewährleistet, dass die Effektivität der Therapie erfasst und alle Nebenwirkungen erkannt und dokumentiert werden, und dass die Erkenntnisse hieraus in das allgemeine Nachsorgekonzept eingebracht werden können. “ Aus Sicht der Genehmigungsbehörde besteht daher die Notwendigkeit zur Erarbeitung fundierter Nachsorgekonzepte für strahlentherapeutische Studien. Im Anschluss an eine ausreichend lange Nachuntersuchung im behandelnden Studienzentrum wäre eine Weiterführung der Verlaufsbeobachtung unter Durchführung von regelmäßigen Telefoninterviews und Versand von Fragebögen denkbar, um den logistischen Aufwand für das Studienzentrum und die Studienteilnehmer (insbesondere bei einem Wohnort in größerer Entfernung zum Studienzentrum) in einem vertretbaren Rahmen zu halten. Es müsste dabei jedoch gewährleistet bleiben, dass den Studienteilnehmern weiterhin regelmäßig ärztliche Nachsorgeuntersuchungen außerhalb des Studienzentrums angeboten werden und dass bei auffälligen Befunden eine Wiedervorstellung im Studienzentrum erfolgt. Alle Nachsorgeergebnisse müssen dabei zentral zusammengefasst und ausgewertet werden.

\section{Ausblick}

In der Vergangenheit haben sich radioonkologische Verfahren entwickelt, die heute weltweit als gängiger Standard gelten, jedoch nie in einer oder mehreren ausreichend großen prospektiven und - falls möglich - randomisierten Studien gegen den vormaligen Goldstandard getestet wurden. Beispiele hierfür sind die stereotaktische Einzeitbestrahlung bzw. die stereotaktische hypofraktionierte Bestrahlung bei kleinen Bronchialkarzinomen oder Oligometastasen sowie die Anwendung eines integrierten Boosts z. B. beim Mammakarzinom. Diese Verfahren wurden quasi „schleichend“ eingeführt. Auch in Zukunft werden neue Therapieverfahren nicht generell in randomisierten Studien getestet werden können. Gerade bei selteneren Tumoren oder Therapieverfahren, die nicht flächendeckend zur Verfügung stehen, scheidet eine Randomisation häufig aus. Bei der zu erwar- tenden Personalisierung der Onkologie anhand von Biomarkern werden zukünftig Patientenkollektive sehr viel kleiner, gleichzeitig aber homogener sein, so dass auch hier klassische randomisierte Studien seltener zum Einsatz kommen werden. Es ist daher zu erwarten, dass prospektive Beobachtungsstudien klinisch und biologisch exakt definierter Patientenkollektive eine zunehmende Rolle spielen. Um genügend Patienten in solche Studien aufnehmen zu können, werden diese meist multizentrisch und oftmals international durchgeführt werden. Hierzu sind entsprechende spezialisierte radioonkologische Datenbanken, in denen die klinischen und biologischen Ausgangsbefunde, Dosispläne und Verlaufsdaten einschließlich der prätherapeutischen und Verlaufsbildgebung aufgenommen werden, von großer Bedeutung und werden derzeit an mehreren Netzwerken weltweit etabliert [7].

Die experimentellen Behandlungskonzepte der großen internationalen randomisierten Studien zur Dosiseskalation beim Prostatakarzinom und beim Mammakarzinom [8-11] haben zu erhöhten frühen und späten Nebenwirkungen der Therapie, gleichzeitig aber auch zu einer verbesserten Tumorwirksamkeit geführt. Insgesamt wurden die Experimentalarme sowohl von der medizinischen und wissenschaftlichen Fachwelt als auch von den Patienten als Verbesserung der Therapie eingeschätzt und stellen derzeit den aktuellen Leitlinienstandard dar. Die Ergebnisse lagen dabei erst viele Jahre nach Abschluss der Behandlungsphase vor. Es wurden mehrere hundert bis mehrere tausend Patienten behandelt, um aussagekräftige Resultate zu erzielen. Wären die genannten Studien aufgrund von erhöhten frühen Nebenwirkungen vorzeitig abgebrochen worden, wäre die positive Nutzen-Risiko-Relation unerkannt geblieben. Zum Nachteil der Patienten wäre die Therapieverbesserung ausgeblieben. Erschwerend kommt hinzu, dass die Nebenwirkungen und Wirkungen einer Strahlentherapie erheblich von der Güte der individuellen Dosisverteilung im Patienten und von individuellen Risikofaktoren abhängen. Diese können anhand des Studienprotokolls nur sehr eingeschränkt oder gar nicht geprüft werden. Daher sind Langzeitbeobachtungen prospektiv untersuchter Patienten von großer Bedeutung für die Weiterentwicklung der Strahlentherapie. Auch in diesem Zusammenhang sind große, spezialisierte radioonkologische Datenbanken, wie sie im letzten Abschnitt beschrieben wurden, notwendig.

Es ist davon auszugehen, dass der Anteil von Patienten, die neben der Strahlentherapie auch eine medikamentöse Antitumortherapie erhalten (neoadjuvant, simultan oder adjuvant) weiter zunehmen wird. Dabei wird eine große Vielfalt neuartiger Ansätze (z. B. klassische Chemotherapeutika, Immuntherapeutika, molekular wirksame Substanzen) zum Einsatz kommen. Darunter werden voraussichtlich auch Medikamente oder therapeutische Verfahren sein, die selbst keine oder nur eine äußerst geringe 
antitumorale Wirkung aufweisen, aber die Strahlenwirkung auf Tumorgewebe oder normale Gewebe modifizieren. Eine Anwendung ionisierender Strahlung, die nach Art und Umfang einem klinisch anerkannten Strahlentherapieverfahren im o. g. Sinne entspricht, muss nicht zwangsläufig gemäß $§ 23$ StrlSchV genehmigungsbedürftig sein, wenn sie im Rahmen einer klinischen Studie in Kombination mit einem neuen medikamentösen Tumortherapieansatz zur Anwendung kommt. Der fachkundige Arzt hat vor Durchführung der Kombinationstherapie anhand des bereits verfügbaren Wissens zu synergistischen Effekten zu beurteilen, ob die für die Stellung der rechtfertigenden Indikation erforderliche Nutzen-Risiko-Abwägung zum Anwendungszeitpunkt mit hinreichender Sicherheit vorgenommen werden kann [12]. Nach Ansicht der Autoren sollten die Ergebnisse aus Studien mit Kombinationstherapien in jedem Fall (d. h. auch bei konventioneller Strahlentherapie) in die entsprechenden radioonkologischen Datenbanken aufgenommen werden, um aussagekräftige Patientenzahlen zu erreichen und den Patientenschutz entsprechend der wissenschaftlich evaluierten Langzeiterfahrungen zu verbessern.

Aus dem Gesagten folgt, dass eine weitere Professionalisierung der Studienplanung und -durchführung in der Radioonkologie sowie die Etablierung spezialisierter radioonkologischer Datenbanken ein wichtiges Ziel für die wissenschaftlichen Fachgesellschaften darstellt. Eine korrekte Bewertung der Genehmigungsbedürftigkeit einer strahlentherapeutischen Anwendung im Vorfeld eines Forschungsvorhabens kann dabei nicht nur unnötige Kosten für die Deckungsvorsorge verhindern, sondern auch eine zeitnahe Bearbeitung genehmigungspflichtiger Anträge begünstigen. Aufgrund der Besonderheiten der radioonkologischen Forschung, die eine spezielle Expertise benötigt, haben das Deutsche Konsortium für Translationale Krebsforschung (DKTK) und das Nationale Zentrum für Radioonkologie (NCRO) mit Unterstützung des Bundesministerium für Bildung und Forschung (BMBF) ein radioonkologisches Medico-Legales-Expertenzentrum in Heidelberg und Dresden aufgebaut. Dieses steht auch den Studienleitern der größten deutschen Studiengruppe, der Arbeitsgemeinschaft Radioonkologie der Deutschen Krebsgesellschaft (ARO), für Beratungen zur Verfügung. DEGRO, BfS und ggf. Landes- und Bundesministerien sowie nationale Netzwerke wissenschaftlich-medizinischer Fachgesellschaften stehen in regelmäßigem Austausch über sich ändernde gesetzliche Rahmenbedingungen (z. B. Umsetzung der Richtlinie 2013/59/EURATOM [13]). Außerdem werden auf Arbeitsebene zwischen Medico-Legalem-Expertenzentrum und BfS auftretende Probleme sowie mögliche Lösungsansätze für spezifische Abläufe und Studien bearbeitet.

\section{Einhaltung ethischer Richtlinien}

Interessenkonflikt M. Simon, M. Habeck, D. Büttner, U. Habeck, T. Nölling, M. Krause, G. Brix, N. Willich, F. Wenz, H. Schmidberger, J. Debus, M. Baumann geben an, dass kein Interessenkonflikt besteht.

Open Access Dieser Artikel unterliegt den Bedingungen der Creative Commons Attribution License. Dadurch sind die Nutzung, Verteilung, und Reproduktion erlaubt, sofern der/die Originalautor/en und die Quelle angegeben sind.

\section{Literatur}

1. Veröffentlicht auf der Homepage des WMA. http://www.wma.net/ en/30publications/10policies/b3/. Zugegriffen: 1. Juli 2015

2. Grundsätze für die Anwendung radioaktiver Stoffe oder ionisierender Strahlung am Menschen in der medizinischen Forschung (Grundsätze nach $\S 41$ StrlSchV) - Stellungnahme der Strahlenschutzkommission (1998) [hrsg. im Auftr. des Bundesministeriums für Umwelt, Naturschutz und Reaktorsicherheit von der Geschäftsstelle der Strahlenschutzkommission beim Bundesamt für Strahlenschutz. Red.: S. Hähnel]. G. Fischer, Stuttgart

3. Pijls-Johannesma M et al (2010) A Systematic methodology review of phase I radiation dose escalation trials. Radiother Oncol 95:135-141

4. Normolle D, Lawrence T (2006) Designing Dose-Escalation trials with late-onset toxicities using the time-to-event continual reassessment method. J Clin Oncol 24:4426-4433

5. Nachsorge für Patienten nach Strahlenbehandlung. Empfehlung der Strahlenschutzkommission, verabschiedet in der 151. Sitzung am 11./12. Februar 1998 (Bundesanzeiger Nr. 144 vom 06.08.1998)

6. Nachsorge als Teil der Qualitätssicherung in der Strahlentherapie zur Überprüfung des Behandlungserfolges. Empfehlung der Strahlenschutzkommission, verabschiedet in der 251. Sitzung der Strahlenschutzkommission am 25.10.2011 (Bundesanzeiger Nr. 38 vom 07.03.2012)

7. Skripcak T et al (2014) Creating a data exchange strategy for radiotherapy research: towards federated databases and anonymised public datasets. Radiother Oncol 113:303-309

8. Bartelink H et al (2001) Recurrence rates after treatment of breast cancer with standard radiotherapy with or without additional radiation. New Engl J Med 345:1378-1387

9. Bartelink H et al (2007) Impact of a higher radiation dose on local control and survival in breast-conserving therapy of early breast cancer: 10-year results of the randomized boost versus no boost EORTC 22881-10882 trial. J Clin Oncol 25:3259-3265

10. Peeters ST et al (2005) Dose-response in radiotherapy for localized prostate cancer: results of the Dutch multicenter randomized phase III trial comparing 68 Gy of radiotherapy with 78 Gy. J Clin Oncol 24:1990-1996

11. Zietman AL et al (2005) Comparison of conventional-dose vs high-dose conformal radiation therapy in clinically localized adenocarcinoma of the prostate: a randomized controlled trial. JAMA 294:1233-1239

12. Kombinationswirkungen Strahlentherapie/medikamentöse Tumortherapie. Empfehlung der Strahlenschutzkommission mit wissenschaftlicher Begründung, verabschiedet in der 264. Sitzung der Strahlenschutzkommission am 21. Oktober 2013 (Bundesanzeiger AT 17.12.2014 B8)

13. Veröffentlicht auf einer Homepage der EU. http://eur-lex.europa.eu/legal-content/DE/TXT/?qid=1431344765979\&uri=CELEX:32013L0059. Zugegriffen: 1. Juli 2015 